\title{
Effect of mixing on enzymatic liquefaction of sago starch
}

\begin{abstract}
The effect of mixing as a function of agitation speed and impeller diameter on the rate and degree of enzymatic liquefaction of sago starch was carried out using a stirred tank reactor with a single Rushton turbine impeller. The performance of the reactor as a mixing device was first examined using different concentrations of carboxymethylcellulose, which exhibited pseudoplastic behaviour similar to that of the solution during the sago starch liquefaction process. A correlation between mixing time (tm) and Reynolds number $(\mathrm{Re})$ in the form of $\mathrm{tm}$ $=\mathrm{bRec}$ is presented; the constants for the correlation depended on viscosity of the fluid. For the two ratios of impeller diameter (Di) to tank diameter (Dt) used, 0.407 and 0.542, agitation speed gave significant influence on both overall rate and degree of liquefaction of sago starch. Mixing time (tm) was independent of impeller diameter used, and correlated well with the overall rate of liquefaction $(\mathrm{P})$ (calculated as the reducing sugar produced divided by time of liquefaction) and expressed as $\mathrm{P}=1.95 \mathrm{tm}-0.362$.
\end{abstract}

Keyword: Enzymatic liquefaction; Sago starch; Mixing; Liquefaction 